\title{
GLUCOSE SYRUP INGESTION AND TEAM PERFORMANCE IN SOCCER
}

\author{
DAVID S. MUCKLE, M.S., F.R.C.S.
}

Radcliffe Infirmary, Oxford

\section{Introduction}

The whole question of nutrition in sport is bedevilled with inconsistencies, faddism and superstition. The popular ingestion of protein (as meat) prior to an event has hallowed precedent in sporting dietetics, although it is impractical and lacks scientific foundation. Carbohydrates and fats serve as energy fuels for muscular activity and the relative contribution of each depends upon their availability from body stores, carbohydrates (as glucose) being utilized preferentially during exercise. Indeed, the consumption of a high carbohydrate diet before a race has been calculated to give a five per cent increase in muscular efficiency, sufficient to increase performance to win the event (Consolazio \& Johnson 1972).

Although the use of a drink containing glucose syrup and mineral salts has been found to improve athletic performance using a cycle ergometer (Brooke, Davies \& Green 1972), treadmill exercise (Green \& Thomas 1972), cycle racing (Brooke \& Davies 1971) and long distance canoeing (Green \& Bagley 1972), little information is available on the effects of glucose ingestion on team performance.

The following study was designed to determine the amount of ball participation, stamina and team performance in soccer players, glucose syrup being given for half the season (twenty matches) while a further twenty matches served as a control. Fortunately, injuries did not interfere with the study - the injury rate being less than three per cent. In fact an intact defence was almost maintained throughout the study.

\section{Method}

Since it has been calculated that soccer players expend 800-1200 kilocals per match, glucose syrup $(420-450$ kilocals, $46 \% \mathrm{w} / \mathrm{v}$, with added mineral salts, Beechams Research Lab.) was given thirty minutes before the event. Emphasis had been placed on a high carbohydrate intake the previous day, with a supplement of 350-450 kilocals of glucose syrup. Thus as a normal dietetic routine was (a) training to exhaustion on Thursday (b) light training on Friday with a high carbohydrate intake (c) glucose syrup on the Saturday match day.

In each match one player was selected by random sampling and his performance assessed by the number of
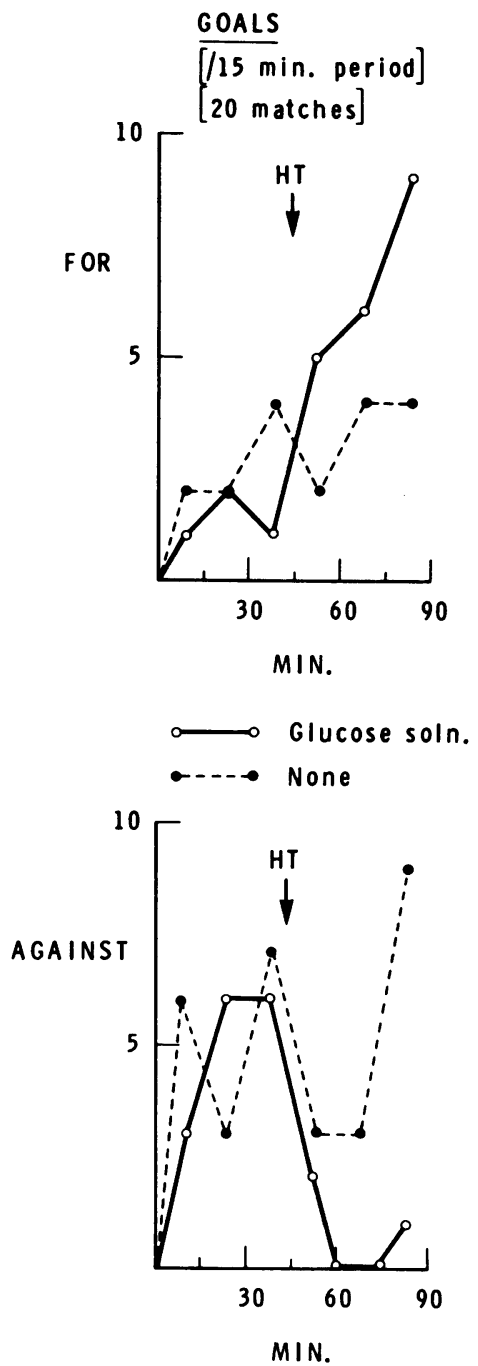

Figures 1 and 2. The goals scored and conceded in each fifteen minute period of the match are plotted against time. $H$. T. indicates half time. Twenty matches in each group, total forty matches.

ball contacts and involvement in play (in minutes) per unit of time (fifteen minute intervals). In no instance was the chosen player injured or substituted. A digital counter and stop watches, with summating second hands (Leonidas) were used in the study.

Team performance was judged by goals 'scored' and 'conceded' in each fifteen minute period of the match. 
Blood glucose samples were obtained during full-scale practice matches (ref. Green 1972).

\section{Results}

Figures 1 and 2 show the effect of glucose and mineral salt ingestion on team performance, - the steady increase in goals scored in the second half contrasts sharply with the marked reduction in goals conceded during the same period (solid lines). However, the control matches show a more uniform distribution of goals 'for' and 'against' (broken lines), with a steep increase in goals conceded during the final fifteen minute period.

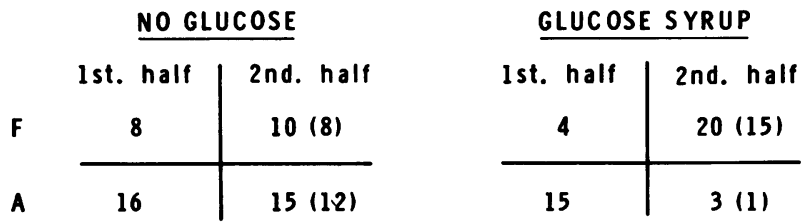

Figures in brackets indicate goals in last $30 \mathrm{mins}$ of $\mathrm{match}$

Figure 3. The total goals scored and conceded in each half are compared (twenty matches in each group).

Figure 3 illustrates certain features of the previous graphs more definitively. When glucose syrup was given the number of goals scored in the second half was double compared to the controls with fifteen goals (out of twenty) being scored in the final half hour of the match. The goals conceded in both halves without glucose, and in the first half with glucose, were almost identical. However, after the ingestion of glucose syrup there was a marked improvement in defensive performance with only one goal conceded during the final thirty minute period of the twenty matches (a total of six hundred minutes).

Figure 4 indicates the effect of glucose and mineral salts on the number of scoring efforts. As in Figure 1 there is a marked improvement in team performance during the last half hour of the match when glucose syrup was given. Indeed, the total number of scoring efforts fell by twenty to fifty per cent in the final fifteen minutes of the match when no glucose was given $(p<$ 0.05).

Figures 5 and 6 . Individual performance was assessed by the number of ball contacts during the fifteen minute intervals throughout the game. The graphs show that the performance reached a peak during the first fifteen minutes in both groups, but whereas the second peak occurred in the fifteen minutes immediately after the interval in the control group, the second peak occurred during the final fifteen minutes of the match when glucose was given. Blood glucose estimations revealed

\section{SCORING EFFORTS}

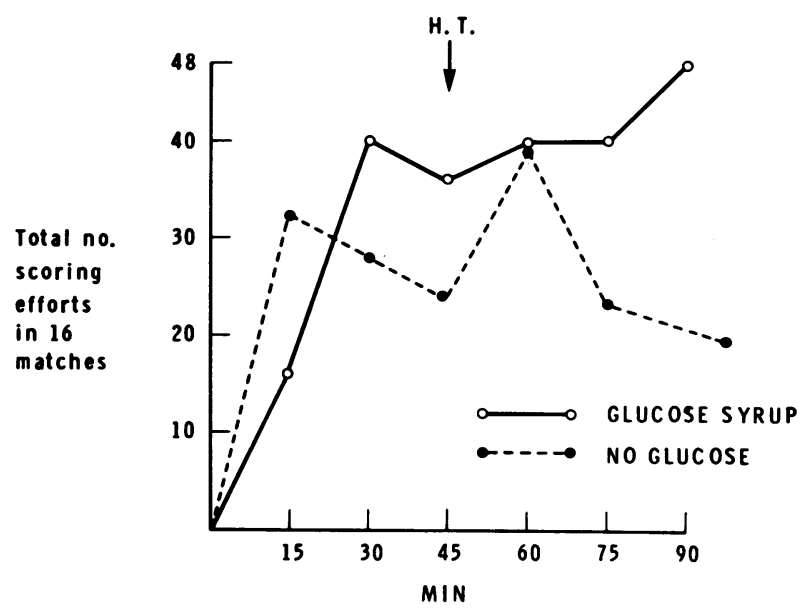

Figure 4. Scoring efforts during each fifteen minute period of the match are plotted against time.

H. T. indicates half time. Six teen matches in each group.

that after the ingestion of glucose the serum level did not fall below the initial reading of $80 \mathrm{mg} / \%$, although the non-glucose controls showed a transient fall in blood glucose during the period of activity.

\section{Discussion}

The ingestion of glucose syrup with added mineral salts prior to soccer improved both team and individual performance as assessed by goals scored and conceded, the total number of scoring efforts and ball-contact. The improvement in team performance was most obvious in the second half, especially the last thirty minutes. Indeed, fifteen goals were scored (sixty per cent) and only one conceded (four per cent) in the final half hour of the match. These results were highly significant $(p<$ $0.001)$ compared to a non-glucose controls and to the results from ten other teams chosen at random in the league. Players not given glucose showed an average twenty to fifty per cent reduction in the number of ball contacts and involvement in play during the final thirty minutes. However, there was a wide variation in individual performance per match, for example, the total number of ball contacts varied between 47 and 122 with the time involved in play fluctuating between 108 and 250 seconds (match play time 57-65 minutes). But in almost every case the peak performance (after glucose) occurred in the first fifteen minutes and the final thirty minutes. By contrast the first half performance, using the above criteria, was not significantly different in both the glucose and non-glucose groups. Although it is now firmly demonstrated that glucose ingestion delays the onset or reduces the fatigue of exercise (Green 1972), the exact mechanism whereby glucose improves performance has not been fully elucidated. 


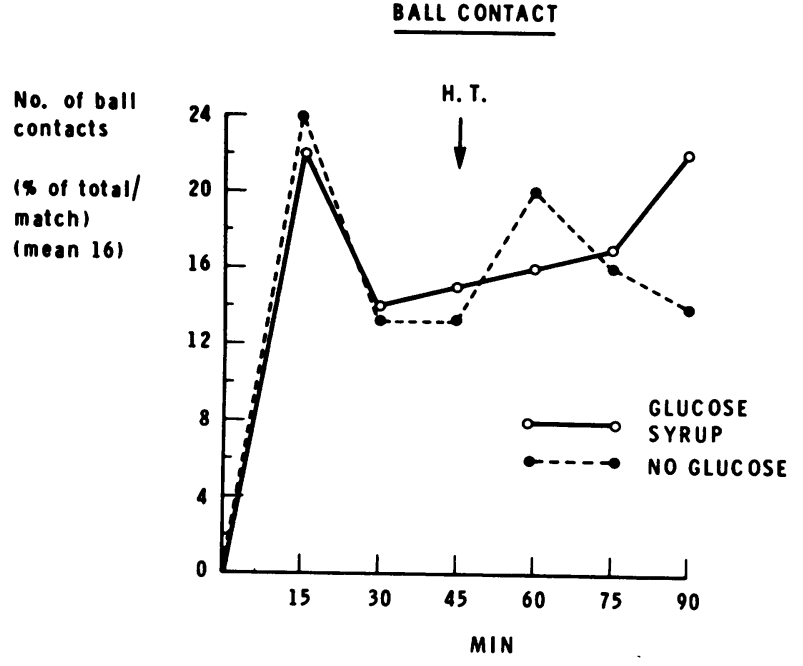

BLOOD SUGAR DURING EXERCISE

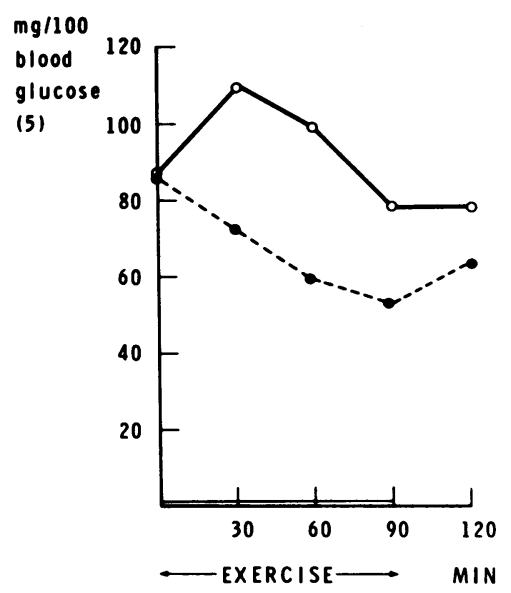

Figures 5 and 6 . The number of ball contacts per fifteen minute period are expressed as a percentage of the total in each match and plotted against time.

The blood sugar level is plotted against time.

In the present trial several factors are involved:

(i) The immediate administration of glucose

(ii) the immediate administration of mineral salts

(iii) the effect of a high carbohydrate diet during the days preceding sporting activity, on the amount of muscle glycogen.

Blood sugar estimations showed that glucose syrup, thirty minutes prior to the event, delayed or reduced the fall in serum levels which are often found during prolonged exercise (Saltin \& Hermansen 1967: Green 1972). However, a transient fall in blood sugar may be followed by a steep rise during activity, and the blood sugar curve often follows a pattern unique for the individual concerned. Thus any a!teration in blood glucose may not necessarily parallel individual performance. Although a close correlation between blood glucose levels and fatigue has not been clearly established, Green (1972) showed an improvement in performance of both submaximal and maximal exercise, in terms of work done and decreased heart rate, occurring concurrently with the elevation of blood sugar.

The importance of carbohydrates as fuel during heavy muscle exercise was shown by Christensen \& Hansen (1939) using respiratory quotient measurements. Their studies also revealed the importance of diet prior to an event. A high carbohydrate diet increased performance two or three fold compared to a high fat diet and they found that during heavy work an increased percentage of carbohydrate was utilised. It is now recognised that the main substrates for energy production for muscular activity are glycogen stores, free fatty acids supplied from the blood and possibly stored muscle triglycerides (Hultman 1967).

However, with prolonged exercise there is a steady reduction in the glycogen content of the working muscle from $1.5 \mathrm{~g} / 100 \mathrm{~g}$. muscle (normal average value) to almost zero, when the subject is completely exhausted. Interestingly, the blood glucose level can be normal while muscle glycogen stores are depleted and the exact mechanism which regulates the uptake of blood glucose by muscle is not known. Recent work has shown that there are two inter-convertible forms of glycogen synthetase, as $D$ form (dependent) and an I form (independent of the presence of glucose-6-P), and lowering the muscle glycogen by exercise increased the I activity from twenty-five to seventy-five per cent. After a period of high carbohydrate intake, there is an overshoot in glycogen synthesis which is not easily explained on the basis of an increased activity of glycogen synthetase $\mathrm{I}$, but could be due to a facilitation of glucose transport into muscle cells (Bergstrom, Hultman \& Roch-Norlund 1972).

An important observation by Saltin \& Hermansen (1967) was that an increase in the glycogen content of the muscles could be produced by a carbohydrate enriched diet and that the effect was more pronounced if prolonged exercise to exhaustion preceded the carbohydrate diet by twenty-four hours. Indeed the glycogen content could be increased two to four fold by rest and a high calorie intake on the day before the event. The ability to perform heavy exercise was found to be directly related to the glycogen stores of the body (Saltin \& Hermansen 1967). While the prior ingestion of 
glucose may keep the blood-sugar at an elevated level, it is probable that the muscle glycogen stores accumulated in the days before activity are of paramount importance in improving athletic ability. For this reason heavy training on preceding days or a prolonged warm-up before the event will utilise glycogen essential for peak performance and thus adversely affect the result.

The ingestion of glucose as well as preventing mild hypo-glycaemia and possible ketosis, has been shown to lessen the exhaustion of strenuous exercise compared to a placebo (Green 1972). However, the part played by the added mineral salts in reducing fatigue has not been investigated independently.

The present study emphasizes the importance of correct dietetics and planned training prior to sporting activity. In general exercise should be tapered off forty-eight hours before competitive effort, and the athlete should rest, taking a high carbohydrate diet. The last meal eaten should be light, high in carbohydrates and can be supplemented with glucose syrup thirty minutes before the event. In this way both individual and team performance can be improved, and a simple rational approach to sporting dietetics maintained.

\section{Acknowledgements}

I would like to thank Mr. L. F. Green and Beecham's Research Laboratory for supplying the glucose drink; Mr. K. Fish for his organisation and administration; Mr. G. Summers and playing staff of Oxford United for their help; and Wendy Cox who assisted with the project.

\section{REFERENCES}

1. BERGSTROM, J., HULTMAN, E., ROCH-NORLUND, A. E. (1972) Sc.J.Clin.Lab.Inv. 29: 231.

2. BROOKE, J., DAVIES, G. J., GREEN, L. F. (1972). Proc.Nut.Soc. (in press).

3. BROOKE, J., DAVIES, G. J. (1971). REPORT Beecham Research. 1971.

4. CONSALAZIO, C. F., JOHNSON, H. L. (1972). Am.J.Clin. Nutr. $25: 85$.

5. ChRISTENSEN, E. H., HANSEN, O. (1939). Skand.Arch.Phys. 81: 152.

6. GREEN, L. F., BAGLEY, R. (1972). Br.J.Sp.Med. 6: 125.

7. GREEN, L. F., THOMAS, V. (1972). Proc.Nut.Soc. 31: no. 1. 5A.

8. HULTMAN, E., BERGSTROM, J. (1967). Acta.Med.Sc. 182 (1): 109.

9. SALTIN, B., HERMANSEN, L. (1967). Symp.Swed.Nut.Found. 5: 32. 